\title{
Stimulus uncertainty does not impair stereopsis
}

\author{
JOSHUA D. STALLER, JOSEPH S. LAPPIN, and ROBERT FOX \\ Vanderbilt University, Nashville, Tennessee 37240
}

\begin{abstract}
The effect of stimulus uncertainty on the stereoscopic resolution of letters was examined for two classes of letters: (1) letters presented stereoscopically as random-element stereograms, and (2) letters presented as two-dimensional physical contours. The variables of stimulus discriminability (stereoscopic vs. physical contours) and stimulus uncertainty (number of alternative letter targets) were combined factorially. Stereoscopically presented letters were more difficult to resolve, but stimulus uncertainty had the same effect for both stereoscopic and physically defined letters. The additivity of these two variables suggests that the perception of stereoscopic forms is an automatic process not impaired by uncertainty about the form to be resolved.
\end{abstract}

Is global stereoscopic resolution impaired by uncertainty about the form to be resolved?

When a naive observer first views a random-element stereogram, perception of the stereoscopic form often requires several seconds, but after additional presentations of the stereogram the time required for perception decreases dramatically. Furthermore, anecdotal reports suggest that stereoscopic form perception may be enhanced when observers are given prior information about the shape of the stimulus to be resolved. Thus, as Julesz (1971) has pointed out, the relatively slow perceptual resolution of random-element stereograms may be sensitive to effects of cognitive processes that are difficult to detect with more conventional stimulus displays.

Previous studies on the resolution of randomelement stereoscopic forms have all used the same basic procedure (Frisby \& Clatworthy, 1975; MacCracken, Bourne, \& Hayes, 1977; MacCracken \& Hayes, 1976; Ramachandran, 1976; Ramachandran \& Braddick, 1973; Saye \& Frisby, 1975). Naive observers are presented with random-element stereograms and asked to report when they perceive a form. The procedure is repeated for 5 to 30 trials, with the same stereogram presented on every trial. The universal finding is that the time to perceive the stereo form decreases over trials, often by more than a factor of 10 . Thus, stereopsis produced by randomelement stereograms is facilitated by practice.

In addition to practice effects, two studies have explored the effects of providing naive observers with prior information about the stereoscopic form to be

This research was supported by NIMH fellowship MH-05499 (J.S.), NSF grant BNS 78-05857 (J.L.), and ONR contract 197-036 (R.F.). J.S. is now at State University of New York at Oswego. resolved. Frisby and Clatworthy (1975) gave three groups of naive observers one of three kinds of information about the form they were about to see. The information was a verbal description, a monocular cue embedded in the stereogram, or a three-dimensional model of the form. All groups showed improvement with practice, but barely more than a control group which received no prior information. The statistical analysis of these data indicated no significant advantage from any of the types of information, although a subsequent reanalysis by Cleveland and Guarino (1978) did reveal a small, yet statistically reliable, benefit from prior information. In a supplementary experiment, Saye and Frisby (1975) found that monocular cues speeded the perception of stereograms with large disparity values, an effect they suggest may be due to the induction of appropriate convergence eye movements.

The possibility that cognitive factors such as prior information may influence stereopsis poses problems for formal models that have been developed to account for global stereopsis (Julesz, 1971; Julesz \& Chang, 1976; Marr \& Poggio, 1976; Sperling, 1970; Sugie \& Suwa, 1977). A basic premise shared by these models is that stereoscopic information is derived from the geometric properties of the stimulus, independently of the observer's knowledge about the disparate contours. Thus, evidence that stereoscopic form resolution varies with the observer's prior knowledge of the contours would seem to demand modification of the hypothesis that extraction of stereoscopic information is solely a geometric affair.

The methodology of the experiments described above, however, does not permit firm conclusions about the specific process that has been influenced by viewing experience or prior information. Because the same stereograms were presented repeatedly, 
knowledge about the shape and location of the target form was confounded with practice effects. Furthermore, the fact that observers were fully aware of the stereoscopic form to be resolved makes it necessary to place great reliance on their ability to maintain a constant response criterion.

The purpose of the present experiments was to determine whether the relative difficulty of resolving stereoscopic forms was invariant with changes in uncertainty about the shape of the target form. In order to measure the effect of uncertainty on stereoscopic resolution, observers were asked to classify target letters in a choice reaction time task in which type of stimulus presentation (stereoscopic vs. physical contours) and stimulus uncertainty (number of alternative letter targets) were systematically varied. Since the resolution and classification of a target form is affected by both the stimulus type (random-element stereograms take longer to resolve than conventional physical-contour forms) and the number of possible targets, the strategy of the present study was to determine whether the combination of these two variables was additive or interactive.

If stereoscopic resolution is not impaired by uncertainty about the target forms, then the effect of increasing the number of alternatives should be the same for both stereoscopic and physical contoursstimulus uncertainty and stimulus type should have independent and additive effects on target classification time. If, however, stereopsis is impaired by stimulus uncertainty, then the resolution of stereoscopic forms should become relatively more difficult as the number of alternative targets increases. Thus, an additive outcome would indicate that the resolution of stereoscopic forms was not impaired by uncertainty, while an interaction would suggest that it was. This contrast between additive and interactive effects is an application of the well-known additive factor logic discussed by Sternberg (1969), although the specific stage model proposed by Sternberg is not at issue here.

At the outset of this study, both empirical and theoretical considerations suggested that stimulus type and stimulus uncertainty might interact. As discussed above, a variety of empirical observations-including perceptual learning, possible effects of prior information, and the slow latency of stereoscopic resolution-indicate that uncertainty might have a greater effect on the detection of stereoscopic forms than on the detection of physical forms. Moreover, there are straightforward theoretical reasons for predicting such an interactive effect.

Consider a model (Anderson, 1973; Lappin, 1978) based on the assumptions: (1) that discrimination between alternative targets derives from numerous stochastically independent perceptual events added to uncorrelated "noise," (2) that different targets are composed of uncorrelated components corresponding to orthogonal vectors, and (3) that successive perceptual events are stochastically independent in time. A model based on these assumptions can predict the well-known linear relationship between reaction time and number of target alternatives (e.g., Sternberg, 1975). Furthermore, this model also predicts an interaction between stimulus type and number of alternatives. If the signal/noise ratio of each component perceptual event depends upon stimulus discriminability (i.e., if the signal/noise ratio were lower for stereoscopic than for physical events), and if the same signal/noise ratios determine the increase in reaction time due to increases in number of targets, then stimulus discriminability and number of targets should have multiplicative (not additive) effects. The increase in reaction time due to increasing number of targets would be greater for the stimuli that are more slowly and less accurately perceived. Such an interaction would be predicted by any model in which the stimulus discriminability independently affects each of the potential target alternatives. Quantitative predictions for a simple illustrative model are derived in the Appendix.

In the following experiments, reaction time varied as a function of the number of target alternatives as well as stimulus type, but these variables did not interact. The model mentioned above predicted that the slope for the stereoscopic contours should be greater than the slope for the physical contours by a factor of about 3.5, but, in fact, the obtained slopes were virtually identical. Thus, the conclusion is that uncertainty about the target form did not impair global stereoscopic resolution.

\section{EXPERIMENT 1}

Stimulus type (stereoscopic vs. physical contours) and number of alternative targets (1 vs. 2 vs. 4 letters per response set) were combined factorially. Response time varied as a function of both variables, but there was no interaction between them.

\section{Method}

Subjects. Five volunteers from the Vanderbilt community served individually. All were paid $\$ 3$ per session and had prior experience with random-element stereograms.

Stimuli and Apparatus. The stimuli were 14 letters of the alphabet (H, Y, W, P, N, Z, L, C, F, K, J, D, X, R) presented individually on a modified color television display (Advent 1000) either as random-dot stereograms or as two-dimensional physical contours.

The random-dot stereograms were generated by a system similar to that described by Fox, Lehmkuhle, and Leguire (1978). In brief, the system generates large matrices $(+30,000$ cells) of red and green dots that are completely replaced every $16 \mathrm{msec}$ with a new randomly selected set of dots. The rapid replacement of dots produces an incoherent apparent movement similar in appearance to dynamic video noise. While the movement does not prevent perception of stereoscopic forms, it does eliminate potential monocular cues. Stereoscopic presentation was implemented by a 
specially designed electro-optical device that has the capability of converting any two-dimensional shape into its stereoscopic counterpart. It does this by programming or specifying the $\mathrm{X}-\mathrm{Y}$ positions in the display where disparity is to be introduced. In this application, the shapes were letters projected by a $35-\mathrm{mm}$ slide projector on a screen that was scanned by the electro-optical programmer. The stereoscopic counterpart of each letter was generated on the color television display. To produce the dichoptic stimulation required for stereoscopic presentation, the anaglyph method (Woodworth, 1938) was used, wherein observers viewed the display through red and green filters (Wratten 58 and 26) which physically segregated the dot matrices so that only one matrix, red or green, stimulated a single eye. The stereoscopic letters, which were $7 \mathrm{deg}$ high and 5 to $9 \mathrm{deg}$ wide, were presented in crossed disparity $(27 \mathrm{~min} 30 \mathrm{sec}$ ), that is, they appeared to lie in front of the projection screen.

The letters presented as two-dimensional physical contours were identical in size and configuration to the stereoscopic letters. Indeed, the physical letters were produced by taking signals from the electro-optical device and selectively suppressing dots electronically so that the final displayed product was a black, dot-free figure against a red-dot background.

Procedure. Observers participated in one practice session followed by three daily $1-\mathrm{h}$ test sessions. Within each session there were six blocks of trials with 32 trials per block. The number of targets per response set-one, two, or four-was systematically varied between trial blocks. Target alternatives were changed every 32 trials and stimulus type was changed every 96 trials. The order of experimental conditions was counterbalanced as much as possible. A single target letter was presented on each trial, and observers responded by classifying it into one of two sets. Half of the letters were designated set 1 and half as set 2 (F vs $K$; D and $X$ vs. $R$ and $J ; Z, C, W$, and $N$ vs. $L, P, H$, and $Y$ ). Stimulus-response mapping was counterbalanced across observers and consistent for each observer throughout testing.

The sequence of events on each trial was as follows: The experimenter signaled that a trial was about to begin; the observer depressed two telegraph keys, and a homogeneous background field of dots appeared (red for the physical targets, red and green for the stereograms); 1 sec later, the test stimulus appeared, superimposed on the background; 4 sec later, the display was turned off. Observers were instructed to release the appropriate telegraph key as soon as they were able to classify the target. Reaction time was measured from the onset of the test stimulus to the onset of the observers' key response. Stimuli were presented in a pseudorandom order and the intertrial interval was about $10 \mathrm{sec}$. The display was binocularly viewed in a dimly lit room from a distance of $12.5 \mathrm{ft}$, and observers wore chromatic filters throughout the entire test session.

\section{Results}

Mean reaction time and mean error percentage values are shown in Figure 1. All observers responded more quickly to physical contours $(\bar{X}=383 \mathrm{msec})$ than to stereoscopic ones $(\bar{X}=445 \mathrm{msec})[\mathrm{F}(1,4)=$ 14.73, $\mathrm{p}<.05$ ]. (Interestingly, some observers with considerable previous stereogram experience responded almost as quickly to the stereograms as to the physical forms - a difference of only about $30 \mathrm{msec}$.)

Response times also varied as a function of the number of possible targets $[F(2,8)=30.26, p<.001]$, and the subjects improved with practice $[\mathrm{F}(2,8)=$ $21.14, \mathrm{p}<.001$ ]. Furthermore, there was an interaction between number of possible targets and practice $[\mathrm{F}(4,16)=3.9, \mathrm{p}<.05]$ due to greater improvement in the four-letter condition than in the twoor one-letter conditions.
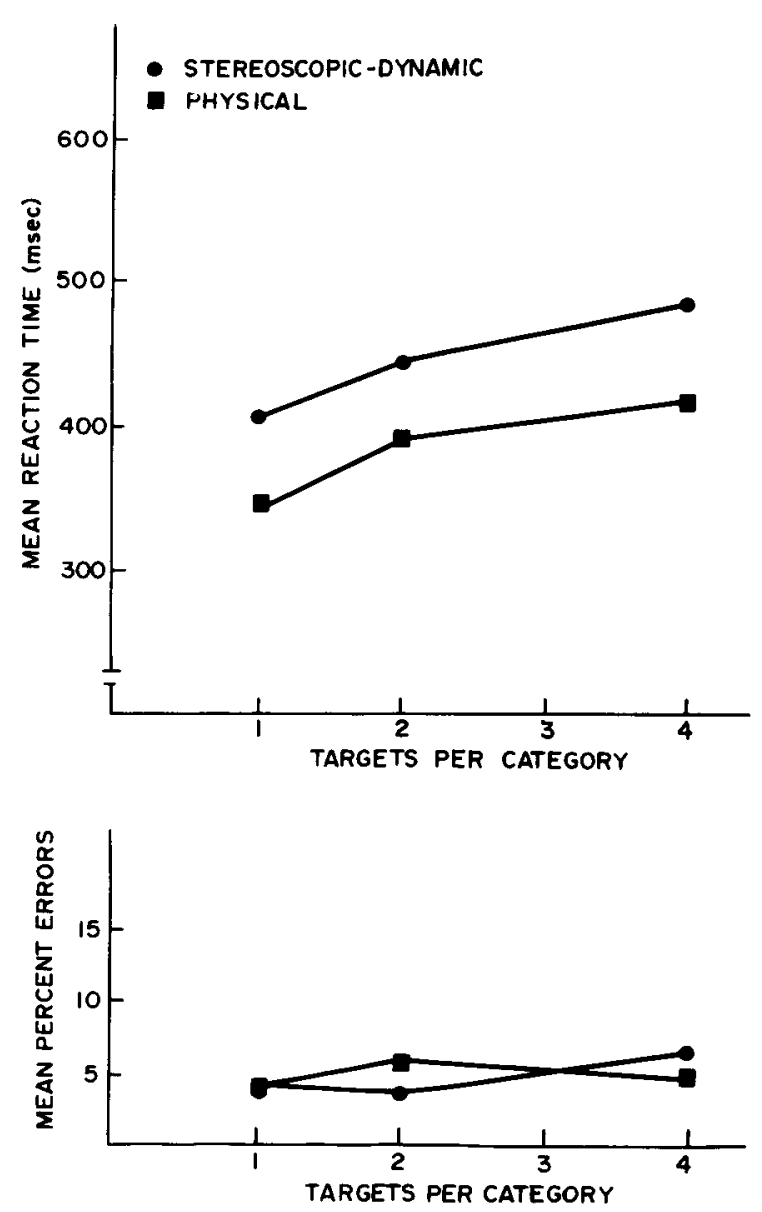

Figure 1. Mean response times and mean percentage errors (Experiment 1) for dynamic stereograms and physical contours at three levels of stimulus uncertainty (1 vs. 2 vs. 4 letters per response set).

There was no interaction between number of target alternatives and stimulus type $(F<1.0)$. Best fitting slope and intercept values for individual subjects are provided in Table 1. Despite the variations in these values, there is little or no indication that the slopes are correlated with the intercepts.

No other reaction time effects were significant (all $p>.25)$. The overall error rate was low (4.6\%), and there were no large error differences between conditions. ${ }^{1}$

\section{EXPERIMENT 2}

In Experiment 1, the effect of the number of alternative targets was the same for both stereoscopicand physical-contour stimuli, a result which indicates that stimulus uncertainty does not impair stereoscopic resolution. In Experiment 2, we tested the generality of this conclusion with two different types of stereograms-static and dynamic. Previous research by Julesz (Julesz \& Kropfl, Note 1) as well as in our own laboratory had indicated that dynamic stereograms were 
Table 1

Best-Fitting Slope and Intercept Values for Individual Subjects

\begin{tabular}{ccccc}
\hline & \multicolumn{2}{c}{ Experiment 1} & \multicolumn{2}{c}{ Experiment 2 } \\
\cline { 2 - 5 } Subject & Physical & Stereoscopic-Dynamic & Stereoscopic-Static & Stereoscopic-Dynamic \\
\hline 1 & $\mathrm{y}=14 \mathrm{x}+296$ & $\mathrm{y}=19 \mathrm{x}+313$ & $\mathrm{y}=42 \mathrm{x}+345$ & $\mathrm{y}=39 \mathrm{x}+388$ \\
2 & $\mathrm{y}=27 \mathrm{x}+288$ & $\mathrm{y}=19 \mathrm{x}+389$ & $\mathrm{y}=24 \mathrm{x}+358$ & $\mathrm{y}=25 \mathrm{x}+403$ \\
3 & $\mathrm{y}=24 \mathrm{x}+401$ & $\mathrm{y}=34 \mathrm{x}+479$ & $\mathrm{y}=50 \mathrm{x}+460$ & $\mathrm{y}=62 \mathrm{x}+824$ \\
4 & $\mathrm{y}=22 \mathrm{x}+344$ & $\mathrm{y}=32 \mathrm{x}+355$ & $\mathrm{y}=53 \mathrm{x}+306$ & $\mathrm{y}=39 \mathrm{x}+359$ \\
5 & $\mathrm{y}=29 \mathrm{x}+316$ & $\mathrm{y}=27 \mathrm{x}+386$ & $\mathrm{y}=49 \mathrm{x}+354$ & $\mathrm{y}=60 \mathrm{x}+437$ \\
Mean & $\mathrm{y}=23 \mathrm{x}+329$ & $\mathrm{y}=26 \mathrm{x}+384$ & $\mathrm{y}=44 \mathrm{x}+365$ & $\mathrm{y}=45 \mathrm{x}+482$ \\
\hline
\end{tabular}

more difficult to resolve than static ones, and it seemed possible that stimulus uncertainty might have a differential effect on perception of these two types of stimuli. Again, however, the effect of uncertainty was the same for both stimulus types, even though the dynamic patterns were responded to more slowly by all observers.

\section{Method}

Subjects. Five new volunteers from the Vanderbilt community served individually. Four received $\$ 3$ per session and one received class credit. None had participated previously in an experiment on stereopsis.

Stimuli, Apparatus, and Procedure. Letter targets were presented as either dynamic or static stereograms. The dynamic stimuli were the same as in Experiment 1-the individual dots appeared to move. In contrast, the position of dots in the static stereograms remained constant throughout a test trial. In all other respects the stimuli, apparatus, and procedure were the same as in Experiment 1.

\section{Results}

Mean reaction time as well as mean error percentage values are presented in Figure 2. All five observers responded more quickly to static stereograms ( $\overline{\mathrm{X}}=$ 466) than to dynamic ones ( $\bar{X}=586$ ), although this difference was not significant by analysis of variance $[F(1,4)=2.98, p<.15]$. Observers also responded more quickly when there were fewer stimulus alternatives $[F(2,8)=44.97, p<.001]$, and performance improved with practice $[\mathrm{F}(2,8)=8.32, \mathrm{p}<.05]$.

There was no interaction between number of stimulus alternatives and stimulus type $(F<1.0)$. Best fitting slopes and intercepts for individual subjects are shown in Table 1. As in Experiment 1, the slopes and intercepts appear to vary independently.

No other effects of the reaction time analysis were significant, all $p>.1$, with the exception of the Stimulus Type by Number of Alternatives by Practice interaction $(\mathrm{p}<.083)$.

The overall error rate was low (3.5\%), and there were no large error differences between conditions.

\section{DISCUSSION}

The present results indicate that increasing the number of stimulus alternatives produces the same increment in reaction time for each of the three stim- ulus types used-dynamic random-element stereoscopic forms, static random-element stereoscopic forms, and physical-contour forms. That is, the functions defining these stimuli had the same slope, and interactions among them were not detected. There were, however, significant differences in discriminability among the stimulus types, as revealed by differences in reaction time (i.e., the reaction time intercept). This pattern of results was anticipated by Sternberg (1967), and suggests two independent pro-
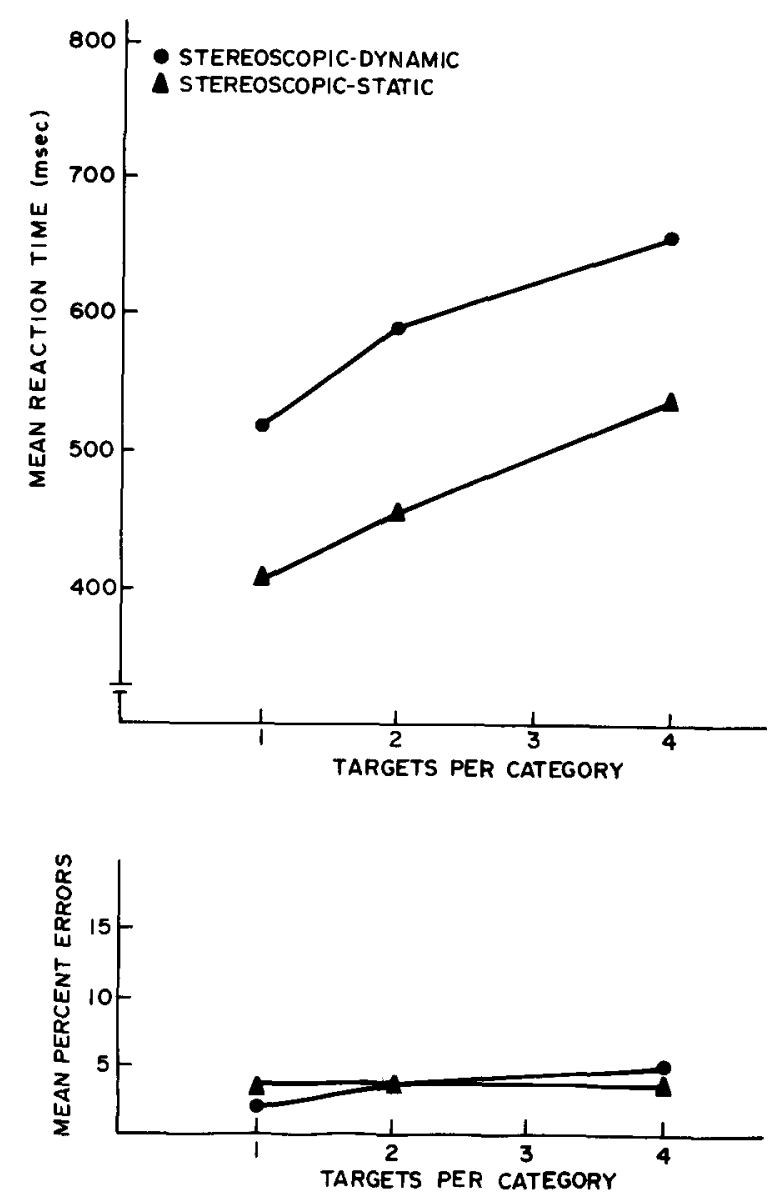

Figure 2. Mean response times and mean percentage errors (Experiment 2) for dynamic and static stereograms at three levels of stimulus uncertainty ( 1 vs. 2 vs. 4 letters per response set). 
cesses for transforming the stimulus into a responsean initial perceptual process that includes stereoscopic resolution, represented by the reaction time intercept, and a subsequent response selection process, represented by the slope of the function. ${ }^{2}$

As discussed in the introduction, functions that yield common slopes can be interpreted to mean that the perceptual processes they represent are not influenced by stimulus uncertainty. Accordingly, the present results demonstrate that target uncertainty exerts little or no influence on the resolution of randomelement stereograms. The primary difference between stereoscopic forms and their physical-contour counterparts is in their initial discriminability. Physical forms are discriminated more rapidly than stereoscopic forms, although the difference, at least for some observers, is not very great (see results of Experiment 1).

The present results also require rejection of the class of models outlined in the introduction which predicts an interaction between stimulus type and number of alternatives. Since the increase in reaction time due to increasing the number of targets was the same for stereoscopic and physical contours in the present study, it must be concluded that stereoscopic presentation and number of alternative targets influence different and presumably temporally separate processes. This conclusion is consistent with the noncognitive character of contemporary models of stereopsis.

It should be noted, however, that strictly speaking, the obtained additivity of effects of stereoscopic presentation and target uncertainty indicates simply that the underlying system is linear. As Lappin (1978) and Taylor (1976) have pointed out, additivity alone does not suffice to determine that experimental variables effect different "stages" or that these stages occur in a particular temporal order. In the present case, however, the absence of an interaction is inconsistent with any model in which the stimulus discriminability independently affects all of the potential target alternatives on each trial. Therefore, stereoscopic resolution must involve a different and presumably earlier process than is affected by the number of target alternatives. The conclusion that stereoscopic presentation and target uncertainty affect different processes does not rest merely on the failure to find a statistically significant interaction, but on the clear absence of the large interaction that would have been expected if both variables affected the same perceptual process (see Appendix).

Finally, as a somewhat parenthetic yet parallel comment, there is a common belief that cognitive factors can directly influence the perception of physicalcontour stimuli. Yet that belief has seldom been demonstrated rigorously, and some work (Lappin \& Uttal, 1976; Staller \& Lappin, 1979; Lappin \& Staller, Note 2) reveals specific instances where it is not true. The present results constitute an additional example in which cognitive factors appear to have no influence on the initial acquisition of perceptual information.

\section{REFERENCE NOTES}

1. Julesz, B., \& Kropfl, W. J. Increased reaction time to dynamic stereograms without monocular cues. Paper presented at the Fourteenth Annual Meeting of the Psychonomic Society, St. Louis, Missouri, November 1-3, 1973.

2. Lappin, J. S., \& Staller, J. D. Prior knowledge does not aid the detection of coherent motion of dynamic random-dot patterns. Paper presented at the Eighteenth Annual Meeting of the Psychonomic Society, Washington, D.C., November 10-12, 1977.

\section{REFERENCES}

Anderson, J. A. A theory for the recognition of items from short memorized lists. Psychological Review, 1973, 80, 417-438.

Cleveland, W. S., \& Guarino, R. The use of numerical and graphical statistical methods in the analysis of data on learning to see complex random-dot stereograms. Perception, 1978, 7, 113-118.

Fox, R., Lehmkuhle, S., \& Leguire, L. E. Stereoscopic contours induce optokinetic nystagmus. Vision Research, 1978, 9, 1189-1192.

Frisby, J. P., \& Clatworthy, J. L. Learning to see complex random-dot stereograms. Perception, 1975, 4, 173-178.

Julesz, B. Foundations of cyclopean perception. Chicago: University of Chicago Press, 1971.

Julesz, B., \& Chang, J. J. Interaction between pools of binocular disparity detectors tuned to different disparities. Biological Cybernetics, 1976, 22, 107-119.

LAPPIN, J. S. The relativity of choice behavior and the effect of prior knowledge on the speed and accuracy of recognition. In F. Restle \& N. Castellan (Eds.), Cognitive theory (Vol. 3). Hillsdale, N.J: Erlbaum, 1978.

LapPIN, J. S., \& UTtal, W. Does prior knowledge facilitate the detection of visual targets in random noise? Perception \& Psychophysics, 1976, 20, 367-374.

MacCracken, P. J., \& Hayes, W. N. Experience and the latency to achieve stereopsis. Perceptual and Motor Skills, 1976, 43, 1227-1231.

MacCracken, P. J., Bourne, J. A., \& Hayes, W. N. Experience and latency to achieve stereopsis: A replication. Perceptual and Motor Skills, 1977, 45, 261-262.

Marr, D., \& Poggio, T. Cooperative computation of stereo disparity. Science, 1976, 194, 283-287.

Pachella, R. G. Interpretation of reaction time in information processing research. In B. Kantowitz (Ed.), Human information processing: Tutorials in performance and cognition. Potomac, Md: Erlbaum, 1974.

Ramachandran, V. S. Learning-like phenomenon in stereopsis. Nature, 1976, 262, 382-383.

Ramachandran, V. S., \& Braddick, O. Orientation-specific learning in stereopsis. Perception, 1973, 2, 371-376.

SAYE, A., \& Frisby, J. P. The role of monocularly conspicuous features in facilitating stereopsis from random-dot stereograms. Perception, 1975, 4, 159-171.

SPerling, G. Binocular vision: A physical and neural theory. American Journal of Psychology, 1970, 83, 461-534.

Staller, J. D., \& Lappin, J. S. Word and nonword superiority effects in a letter detection task. Perception \& Psychophysics, $1979,25,47-54$.

STERnBe RG, S. Two operations in character recognition: Some evidence from reaction-time measurements. Perception \& Psychophysics, 1967, 2, 45-53. 
Sternberg, S. The discovery of processing stages: Extensions of Donders' method. In W. G. Koster (Ed.), Attention and performance II. Acta Psychologica, 1969, 30, 276-315.

Sternberg, S. Memory scanning: New findings and current controversies. Quarterly Journal of Experimental Psychology, 1975, 27, 1-32.

Sugie, N., \& Suwa, M. A scheme for binocular depth perception suggested by neurophysiological evidence. Biological Cybernetics, 1977, 26, 1-15.

TAYLOR, D. A. Stage analysis of reaction time Psychological Bulletin, 1976, 83, 161-191.

Woodworth, R. S. Experimental psychology. New York: Holt, 1938.

\section{NOTES}

1. In a preliminary experiment 4 observers were tested with physical vs. dynamic contours and 1 vs. 4 letters per response set. Though the response measure and stimulus letters were somewhat different than in Experiment 1, the results were essentially the same. There was a significant effect of stimulus type $(p<.05)$ and number of alternatives $(p<.001)$, but no interaction between these two variables, $F<1.0$.

2. This conclusion does not depend upon acceptance of the complete model proposed by Sternberg (1969). Specifically, one need not assume that the linear dependence of reaction time on the number of alternative targets indicates a serial process such as memory scanning. An alternative model (e.g., Anderson, 1973; Lappin, 1978) attributes such effects to the statistical characteristics of the stimuli without postulating any limitation on perception or memory. The basic assumptions of this model are outlined in the Introduction and in the Appendix. Briefly, such a model predicts the well-known linear relation between number of alternative targets and reaction time in the Sternberg binary classification task without postulating any processing limitation such as serial memory scanning. However, the same simple model will not account for the additivity of stimulus discriminability and number of alternative targets (Sternberg, 1967), contrary to the suggestion of Lappin (1978).

(Received for publication April 9, 1979;

Revision accepted January $17,1980$. )

\section{APPENDIX}

In this appendix, we briefly review the rationale of a simple model to account for the well-known linear relation between RT and the number of alternative targets and to predict the relation between the slopes of these functions for physical and stereoscopic targets. This model is developed and discussed in more detail by Lappin (1978) and in a slightly different form by Anderson (1973). The application to the present problem of predicting the change in slope associated with a change in the mode of stimulus presentation is new, and the present appendix corrects an incorrect claim by Lappin (1978) that changing the stimulus discriminability should have no effect on the slope of the relation between $\mathrm{RT}$ and number of targets.

The basic assumptions are: (1) Perceptual observations of a stimulus consist of large numbers of independent components added to random noise, with noise generated by a Poisson process with independent and identically distributed values at different points in time and space. (2) Different targets are compos ${ }^{-1}$ of uncorrelated components, corresponding to orthogonal vectors. (3) Successive perceptual events are independently and identically distributed. (4) The RT for classifying targets is equal to a constant plus the duration of observation required for the perceptual information to reach a criterion signal/noise ratio. In other words, performance is presumed to be limited by a stationary noise process with variance directly proportional to the observation interval.

The signal/noise ratio is equal to the signal amplitude (the expected value of the perceptual observation) divided by the standard deviation of the noise; in other words, the signal/noise ratio equals $\mathrm{d}^{\prime}$, the signal detection theory measure. Suppose that the observation interval is multiplied by a factor of $\mathrm{T}$; then the signal amplitude will increase by the same factor, while the standard deviation of the noise increases by $\sqrt{T}$, resulting in a net change in the signal/noise ratio by $\sqrt{T}$. Thus, if $d^{\prime}(T)$ is the signal/ noise ratio for an observation interval of $\mathrm{T}$ units of time, then

$$
d^{\prime}(T)=\sqrt{T} d^{\prime}(1) .
$$

In other words, the signal/noise ratio, $d^{\prime}$, is directly proportional to the square root of the temporal duration of the observation interval.

Next, consider how $d^{\prime}$ varies with the number of equally likely alternative targets. By hypothesis, we assume that different targets are uncorrelated. If the observer attends equally to each of $M$ alternatives but the actual target is only one of these, then the variance of the noise will be $M$ times greater than in the case with only one alternative. Thus, if the number of targets is increased by a factor of $\mathbf{M}$, then the signal/noise ratio will be reduced by a factor of $1 / \sqrt{\mathrm{M}}$. This reduction in the signal/noise ratio, associated with an increase in the number of targets, can be exactly compensated by a corresponding increase in the observation interval. That is, if $\mathbf{M}$ is the number of equally likely targets and $d^{\prime}(M ; T)$ is the signal noise ratio for $M$ alternatives and an observation interval of $\mathrm{T}$ time units, then

$$
\begin{aligned}
d^{\prime}(M ; T) & =(1 / M)^{1 / 2} d^{\prime}(1 ; T) \\
& =T^{1 / 2} d^{\prime}(M ; 1) \\
& =(T / M)^{1 / 2} d^{\prime}(1 ; 1) .
\end{aligned}
$$

Thus, if $M=T=k$, then $d^{\prime}(k ; k)=d^{\prime}(1 ; 1)$.

Now it is easy to see that if RT is equal to the observation interval plus a constant, then RT will also be a linear function of the number of alternative targets. That is, if RT = $a+T$, where $a$ is a positive constant, then, by substituting for $T$ and squaring both sides of the equation, we can write

$$
\begin{aligned}
d^{\prime 2}(M ; T) & =(R T-a) / M\left[d^{\prime 2}(1 ; 1)\right] \\
R T & =a+M\left[d^{\prime}(M ; T) / d^{\prime}(1 ; 1)\right]^{2} .
\end{aligned}
$$

Equation 3 expresses the hypothesis that the slope of the RT vs. $M$ relationship increases with the signal/noise ratio. As the observer's response criterion (defined on the signal/ noise ratio) increases, the slope also increases. Qualitative support for this hypothesis may be found in a paper by Pachella (1974), who showed that when the speed/accuracy 
tradeoff is varied, the slope of the RT vs. $M$ relationship increases with the accuracy and latency of the responses. Similar results have been observed in our own laboratory (unpublished data). In order to equate the response criteria for different values of $\mathbf{M}$ and to eliminate this variable response criterion from the equation, let us arbitrarily set the variable numerator equal to the denominator. From Equation 2, we know that $d^{\prime}(M ; T)=d^{\prime}(1 ; 1)$ if and only if $\mathrm{M}=\mathrm{T}$, where $\mathrm{T}$ is measured in arbitrary time units. If $\mathrm{T}$ is expressed in milliseconds, let the signal/noise ratio associated with one unit of $M$ be equal to that associated with b msec of observation, that is, $d^{\prime}(M ; b$ msec $)=d^{\prime}(1 ; 1 \mathrm{msec})$. Thus,

$$
\begin{aligned}
d^{\prime}(M ; b) & =d^{\prime}(1 ; 1) \\
& =\sqrt{b} d^{\prime}(M ; 1)
\end{aligned}
$$

and

$$
R T=a+M\left[d^{\prime}(M ; b) / d^{\prime}(1 ; 1)\right]^{2},
$$

which gives the linear relation between $R T$ and $M$ :

$$
\mathbf{R T}=\mathbf{a}+\mathbf{b} \mathbf{M}
$$

To see how stereoscopic presentation will affect this relationship, suppose that the visibility or signal/noise ratio of the stereoscopic targets is some fraction of that for physical targets-say,

$$
\left[d^{\prime}(M ; T ; \text { stereo. }) / d^{\prime}(M ; T ; \text { phys. })\right]^{2}=1 / c \text {, }
$$

where $c \geqslant 1.0$. Such decrements in signal/noise ratio can be compensated by increasing the observation interval:

$$
\begin{aligned}
\mathrm{d}^{\prime 2}(\mathrm{M} ; \mathrm{T} ; \text { phys. }) & =c \mathrm{~d}^{\prime 2}(\mathrm{M} ; \mathrm{T} ; \text { stereo. }) \\
& =\mathrm{d}^{\prime 2}(\mathrm{M} ; \mathrm{c} \mathrm{T} \text {; stereo. })
\end{aligned}
$$

Thus, following the same procedure as before to set the response criterion (signal/noise ratio) for the stereoscopic targets equal to that for the physical targets, we have

$$
\begin{aligned}
\mathbf{R T} & =a+b \mathbf{M}\left[d^{\prime}(1 ; c ; \text { stereo. }) / d^{\prime}(1 ; 1 ; \text { physical })\right]^{2} \\
& =a+b c \mathbf{M} .
\end{aligned}
$$

So, if stereoscopic presentation reduces the same signal/ noise ratio that is involved in the reaction time for selecting an identifying response to the perceived target, then more time will be required to compensate for increasing numbers of alternative stereoscopic targets. The predicted increase in slope, $c$, is easily estimated from the data in the $M=1$ condition:

$$
\begin{aligned}
\operatorname{RT}(\text { stereo. })-\operatorname{RT}(\text { phys. }) & =(a+b c M)-(a+b M) \\
& =b M(c-1) .
\end{aligned}
$$

When $\mathbf{M}=1$,

$$
c=1+[\text { RT(stereo.) }- \text { RT(phys.) }] / \text { b. }
$$

In Experiment 1, the slope b of the function relating RT to $M$ in the physical presentation was $23 \mathrm{msec}$. When $M=1$, the average difference in $\mathrm{RT}$ for the stereoscopic and physical presentations was $61 \mathrm{msec}$. Thus, the predicted slope for the stereoscopic condition should have been greater than the slope for the physical condition by a factor of $c=3.63$. In fact, the slope for the stereoscopic condition was $26 \mathrm{msec}$, not $89 \mathrm{msec}$.

In Experiment 2, the slopes for the static and dynamic stereograms were 44 and $45 \mathrm{msec}$, respectively, though by the above equations the slope for the dynamic stereograms should have been $157 \mathrm{msec}(\mathrm{c}=3.59)$.

Obviously, the predicted increases in slope are inconsistent with the obtained data; some aspect of the model is incorrect. The problem appears to lie in the assumption that stimulus type affects the same signal/noise ratio as that which determines the slope of the relation between RT and $M$. The absence of an interaction between discriminability and uncertainty is inconsistent with any model in which the discriminability has a stochastically independent ef fect on each trial on the identification of all of the potential alternative targets. The conclusion is that stimulus type must affect a different source of confusability than that involved in the categorization of the targets. 